\title{
Unravelling a new many-body large-hole polaron in a transition metal oxide that promotes high photocatalytic activity
}

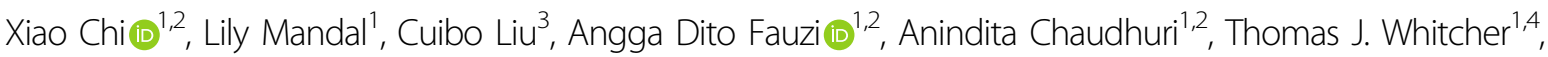 \\ Hariom Kirit Jani $\mathbb{1}^{1,5}$, Zhongxin $\mathrm{Chen}^{3}$, Shibo $\mathrm{X}^{6}{ }^{6}$, Caozheng Diao $\mathbb{E}^{2}$, Muhammad Avicenna Naradipa $\mathbb{E}^{1,2}$, \\ Xiaojiang Yu $\mathbb{0}^{2}$, Ping Yang ${ }^{2}$, Antonio Helio Castro-Neto ${ }^{1,4}$, Mark B. H. Breese ${ }^{1,2}$, Kian Ping Loh $\mathbb{1}^{3}$, \\ Thirumalai Venky Venkatesan ${ }^{1,5,7}$ and Andrivo Rusydi $\mathbb{B}^{1,2,4,5}$
}

\begin{abstract}
A many-body large polaron, which is important for both fundamental physics and technological applications, has been predicted to occur in bismuth vanadate $\left(\mathrm{BiVO}_{4}\right)$. Herein, using a combination of high-resolution spectroscopic ellipsometry, $\mathrm{X}$-ray absorption spectroscopy at the $\mathrm{V} L_{3,2^{-}}$and $\mathrm{O} K$-edges, and high-resolution $\mathrm{X}$-ray diffraction supported by theoretical calculations, we reveal a new many-body large-hole polaron in W-doped $\mathrm{BiVO}_{4}$ films and the interplay of the large-hole polaron and indirect bandgap when determining the photocatalytic activity. With various $\mathrm{W}$ doping concentrations and temperatures, anomalous spectral weight transfers in the complex dielectric function are observed, revealing electronic correlations, particularly the on-site Coulomb interactions of $\mathrm{O} p\left(\mathrm{U}_{p p}\right)$ and $\vee d\left(\cup_{d d}\right)$, and screening in $\mathrm{BiVO}_{4}$. Due to the distortion of $\mathrm{BiO}_{8}$ dodecahedra and $\mathrm{U}_{d d}, \mathrm{Bi} 6 s$ is lifted to the top of the valance band, which results in the formation of an indirect bandgap and a large-hole polaron. The large-hole polaron is found to form as a localized midgap state, consisting of $\mathrm{O} p$ hybridized with the $\mathrm{V} d$ and $\mathrm{Bi} s p$ orbitals, and this is important when determining the high photocatalytic activity of $\mathrm{BiVO}_{4}$. Our results show the importance of the interplay among the charge, orbital, and lattice degrees of freedom in forming the many-body large-hole polaron, which improves the conductivity and results in a transition metal oxide with high photocatalytic activity.
\end{abstract}

\section{Introduction}

Understanding how an electron travels through a solid and its subsequent distortion of the surrounding lattice ${ }^{1}$, also known as a polaron ${ }^{2}$-a manifestation of many-body interactions-has been of great interest since the early 1930s. Currently, polarons are believed to play important roles in determining various exotic properties in strongly

\footnotetext{
Correspondence: Xiao Chi (chixiao1016@gmail.com) or Kian

Ping Loh (chmlohkp@nus.edu.sg) or Thirumalai Venky Venkatesan

(venky@nus.edu.sg) or Andrivo Rusydi (andrivo.rusydi@nus.edu.sg)

'Department of Physics, National University of Singapore, 117576 Singapore,

Singapore

${ }^{2}$ Singapore Synchrotron Light Source, National University of Singapore, 5

Research Link, 117603 Singapore, Singapore

Full list of author information is available at the end of the article.

These authors contributed equally: Xiao Chi, Lily Mandal, Cuibo Liu
}

correlated electron systems, such as superconductivity ${ }^{3-5}$, metal-insulator transition $^{4,6,7}$, and ferroelectricity ${ }^{8,9}$. Concurrently, photocatalytic activities such as water splitting have been increasingly studied because of the rich fundamental science involved and their potential applications. Indeed, polarons have been theoretically argued to have significant roles in photocatalytic activities $^{10-12}$, which have been explored for the solar-powered splitting of water to generate oxygen/hydrogen ${ }^{13-22}$. Oxide semiconductor-based materials, such as $\mathrm{BiVO}_{4}{ }^{19,23}$, $\mathrm{TiO}_{2}{ }^{15,22}, \mathrm{SrTiO}_{3}{ }^{24}$ and $\mathrm{WO}_{3}{ }^{25}$, are thought to be promising photocatalytic candidates, considering their high photocatalytic activities in converting solar irradiation to energy by facilitating the absorption of photons to generate photoelectrons and holes. However, the mechanism 
behind the high photocatalytic activities in oxides is lacking.

It has been theoretically predicted that photoinduced electrons and holes can be separated by a large polaronic state, as such a large polaron may be crucial to avoid recombination, consequently diffusing through the crystal and culminating in a photocatalyzed reaction. However, the carrier transport properties of transition metal oxides are typically low, especially when they are compared to those of III-V semiconductors or even silicon. Transition metal oxides, even with electron/hole doping, still possess low conductivity due to the localization of charge carriers, which form small polarons ${ }^{26}$. Of particular interest is $\mathrm{BiVO}_{4}$, which has tunable bandgaps, substantial absorption in the visible spectrum, and a favorable conduction band (CB) edge position near the thermodynamic hydrogen evolution potential ${ }^{19,23,27-30}$. Previous studies have suggested that the photocatalytic performance of pure $\mathrm{BiVO}_{4}$ is not as good as expected, and it is suspected that the conduction mechanism is charge carrier diffusion via small-electron polaron hopping ${ }^{31-33}$. Interestingly, after doping molybdenum (Mo) or tungsten (W), the conductivity of $\mathrm{BiVO}_{4}$ obviously increases, and the photocatalytic activation is significantly enhanced ${ }^{34,35}$, which cannot be explained by the small-electron polaron hopping mechanism. Previous theoretical studies have suggested that a large-hole polaron may be formed in monoclinic $\mathrm{BiVO}_{4}\left(\mathrm{~m}-\mathrm{BiVO}_{4}\right)$ promoting its candidacy as a good photocatalyst for solar water splitting ${ }^{12,36}$. However, the large-hole polaron in the $\mathrm{BiVO}_{4}$ system has yet to be experimentally observed, and our understanding of large polaronic state formation in the photoexcited state and how it affects photoconversion efficiency, in general, are still lacking, especially since the large polaron is a manifestation of many-body interactions.

An important factor for solar energy conversion is that photocatalysts require a small bandgap in order to absorb a wider range of visible light. For instance, theoretical calculations have shown that after decreasing the bandgap from 2.5 to $2.3 \mathrm{eV}$, the maximum photocurrent $\left(J_{\max }\right)$ may increase $40 \%$, assuming $100 \%$ incident photon-to-current conversion efficiency (IPCE), for photons with energy exceeding the bandgap ${ }^{37,38}$. As a first step in energy conversion, electron-hole pairs generated by light absorption need to be separated and transferred to the surface of the semiconductors. It has been theoretically suggested that an indirect band gap-assisted polaron may also be needed for charge separation ${ }^{28,29}$, as both charge absorption and recombination involve not only photons but also phonon (lattice) effects. This charge-lattice interaction can reduce electron-hole recombination and increase the charge diffusion length.

In this work, we reveal a new many-body large-hole polaron along with strong electronic correlations and screening that show that the interplay of the large-hole polaron and indirect bandgap is important in determining the photocatalytic activity of $\mathrm{BiVO}_{4}$ films. The $\mathrm{BiVO}_{4}$ films are grown using pulsed laser deposition with increasing tungsten (W) doping (from $0 \%$ to $5 \%$ ) and various crystal phases. We specifically choose W-doped $\mathrm{BiVO}_{4}$ for the following reasons. First, it belongs to a strongly correlated electron system, which is essential to generate a manybody polaronic state. Second, from X-ray photoemission data (Supplementary Fig. S1), W is multivalent $\left(\mathrm{W}^{5+}\right.$ and $\mathrm{W}^{6+}$ ), which helps to create a hole state while maintaining charge neutrality. Third, W can also modify the crystal structure $^{39}$. Because of these factors, $W$ doping assists in stabilizing vanadium vacancies $\left(\mathrm{V}_{\mathrm{v}}\right)$ and introducing holes, simultaneously creating local lattice distortions; all the above together can generate a hole polaron in the $\mathrm{W}_{-} \mathrm{BiVO}_{4}$ system (Supplementary Fig. S2). This is also supported by the fact that $\mathrm{BiVO}_{4}$ has a lower formation energy for the creation of vanadium vacancies $\left(\mathrm{V}_{\mathrm{v}}\right)$ than that of oxygen/bismuth vacancies $\left(\mathrm{O}_{\mathrm{v}} / \mathrm{Bi}_{\mathrm{v}}\right)^{40}$. These cation vacancies created by substitution with multivalence elements have been observed in other oxides ${ }^{41,42}$. We use a comprehensive method comprising high-resolution spectroscopic ellipsometry (SE), X-ray absorption spectroscopy (XAS) at the $\mathrm{V} L$ - and $\mathrm{O} K$-edges, $\mathrm{X}$-ray photoemission spectroscopy, and X-ray diffraction supported by theoretical calculations to experimentally determine the manybody large-hole polaron, electronic and crystal structures, and photocatalytic nature of $\mathrm{BiVO}_{4}$. SE is a powerful technique to directly and simultaneously probe large-hole polarons and the associated electronic and optical properties. Supported by XAS, the large-hole polaron is found to form a new midgap state, the band gap becomes indirect, and importantly, there is a large spectral weight transfer over a broad energy range as a function of $\mathrm{W}$ doping in $\mathrm{BiVO}_{4}$. Supported by theoretical calculations, the spectral weight transfer, which is a fingerprint of strong electronic correlations, is particularly due to on-site Coulomb interactions in $\mathrm{O} p\left(\mathrm{U}_{p p}\right)$ and $\mathrm{V} d\left(\mathrm{U}_{d d}\right)$, induced by holes. The many-body large-hole polaron consists of $\mathrm{O}$ $p$ states hybridized with $\mathrm{V} d$ and $\mathrm{Bi} s p$ states due to the distortion of $\mathrm{BiO}_{8}$ dodecahedra, and the indirect bandgap is attributed to the interband transitions from $\mathrm{Bi} 6 s$ to $\mathrm{V}$ $3 d$ assisted by the large-hole polaron. Interestingly, the $1 \%$ $\mathrm{W}$-doped monoclinic $\mathrm{BiVO}_{4}$ shows the most prominent large-hole polaron and indirect bandgap due to electronic correlations and screening, which decreases $\mathrm{O} 2 p$ - $\mathrm{Bi} 6 s / 6 p$ hybridization, lifts $\mathrm{Bi} 6 p / 6 s$ orbitals to the top of the valence band and improves conductivity; thus, $\mathrm{BiVO}_{4}$ exhibits high photocatalytic water splitting performance. Furthermore, the large-hole polaronic states have a weak binding energy, which preserves the band-like transport of electrons/holes and strongly suppresses electron-hole recombination. 


\section{Materials and methods}

\section{Sample preparations}

The W-doped $\mathrm{BiVO}_{4}$ ceramic target was synthesized by a solid-state reaction using $\mathrm{Bi}_{2} \mathrm{O}_{3}, \mathrm{~V}_{2} \mathrm{O}_{5}$ and $\mathrm{WO}_{3}$ (High Purity Chemicals, Japan) at nominal ratios to obtain $0-5 \%$ W weight doping. The predecessors were mixed evenly and calcined at $900{ }^{\circ} \mathrm{C}$ for $5 \mathrm{~h}$ and pressed as a target. $\mathrm{BiVO}_{4}$ thin films with $0 \%, 0.5 \%, 1 \%, 2 \%$ and $5 \% \mathrm{~W}$ doping were epitaxially deposited on yttrium-doped zirconium oxide (YSZ) (001) substrates by pulsed laser deposition (PLD). Laser ablation was performed at a repetition rate of $2 \mathrm{~Hz}$ and an energy density of $80 \mathrm{~mJ} / \mathrm{cm}^{2}$ with a $248 \mathrm{~nm}$ $\mathrm{KrF}$ excimer laser. The substrate temperature was $600{ }^{\circ} \mathrm{C}$ in this work, and the $\mathrm{O}_{2}$ partial pressure was kept at 100 mbar during growth. The crystal structures and epitaxial strain of the $\mathrm{BiVO}_{4}$ films were characterized by synchrotron-based X-ray diffraction techniques on the XDD beamline at the Singapore Synchrotron Light Source (SSLS). The $\theta-2 \theta$ scan of all samples is shown in Fig. S3, and the diffraction peaks are all noted.

\section{Photocatalytic measurements}

The photocatalytic experiments were carried out in a $200 \mathrm{ml}$ vessel filled with $0.2 \mathrm{M} \mathrm{Na} \mathrm{N}_{2} \mathrm{~S}$ and $0.3 \mathrm{M} \mathrm{Na}_{2} \mathrm{SO}_{3}$ solution. The prepared $\mathrm{BiVO}_{4}$ film was immersed in the mixed solution with light irradiation. The amount of $\mathrm{H}_{2}$ gas involved was determined by taking $100 \mu \mathrm{L}$ of gas from the headspace of the cell using a syringe and injecting it into the gas-sampling loop of the gas chromatograph every three hours. The gas chromatograph was equipped with a packed MolSieve 13X column. Helium was used as the carrier gas. A helium ionization detector was used to quantify the hydrogen concentration.

\section{First-principles calculations}

The electronic structure of $\mathrm{BiVO}_{4}$ was calculated using density functional theory (DFT) as implemented in the Quantum ESPRESSO package ${ }^{43}$. The optimized normconserving Vanderbilt pseudopotential ${ }^{44}$ was employed for the exchange-correlation functional. The system was modeled using a unit cell consisting of 24 atoms in a monoclinic structure. An electron was removed from the system to mimic the hole-doped condition. Charge neutrality was ensured by inserting a compensating jellium background into the system. The kinetic energy cutoff was set to $75 \mathrm{Ry}$. The structure was optimized until all forces of the constituent atoms were less than $10^{-3} \mathrm{Ry} / \mathrm{Bohr}$, and then a self-consistent calculation was performed with an energy threshold of $10^{-8}$ Ry. A Monkhorst-Pack $k$-point grid of $6 \times 6 \times 6$ was used for structural optimization, while a denser $k$-point grid of $12 \times 12 \times 12$ was utilized to calculate the density of states of the system. The optimized lattice parameters were $a=7.151 \AA, b=11.358 \AA$, $c=5.056 \AA$ and $\beta=135.03^{\circ}$, which agreed with previously reported results ${ }^{11}$. To address the effect of strong electron correlation in the $\mathrm{O} 2 p$ and $\mathrm{V} 3 d$ orbitals, the Hubbard $U$ parameter was incorporated within the DFT $+U$ scheme $^{45-47}$.

\section{Results and discussion}

W-doped $\mathrm{BiVO}_{4}(0 \%, 0.5 \%, 1 \%, 2 \%$ and $5 \% \mathrm{~W})$ is epitaxially deposited on yttrium-doped zirconium oxide (YSZ) (001) substrates (Sample preparations). The crystal structures with reciprocal-space mappings (RSMs) are characterized by high-resolution synchrotron-radiation $\mathrm{X}$-ray diffraction measurements. We selected $H K$-plane mapping near the $(-204)$ diffraction to monitor the strain texture in-plane, and the results are shown in Fig. 1. The pure $\mathrm{BiVO}_{4}$ RSM displays a symmetric fourfold diffraction spot, where two peaks are shifted up and others are shifted down equally along both the $H$ and $K$ directions (Fig. 1a). The symmetric shift of the $(-204)$ diffraction spot implies that there are two different lengths of the inplane axes $a$ and $b$, which are orthogonal to each other to form an orthorhombic (O) phase, and the fourfold symmetric diffraction spot signifies that the crystal is twinned. Based on the diffraction positions, the lattice parameters are determined to be $a=5.101 \pm 0.003 \AA, b=5.192 \pm$ $0.001 \AA$, and $c=11.697 \pm 0.001 \AA$ (calculated by $L$-scan in Supplementary Fig. S3), in which the average diagonal length of the lattice in the $a b$ plane is $7.275 \AA$, which is close to the $7.278 \AA$ for the yttrium-doped zirconium oxide (YSZ) substrate. In contrast, the twin weakens as a function of $\mathrm{W}$ doping, as shown in Fig. 1b, c.

The lightly introduced dopant $\mathrm{W}$ significantly changes the distortion in the $\mathrm{BiVO}_{4}$ lattice, and the diffraction spots deviate from the symmetric lines, indicating that the $a b$ axials are no longer perpendicular and that the twin phase becomes a monoclinic (M) structure (as shown in Fig. 1c for $1 \% \mathrm{~W}$ doping). The twin disappears when $\mathrm{W}$ doping is above $2 \%$ (Fig. 1d, e), and the $\mathrm{BiVO}_{4}$ film is fully strained in the $a b$ plane, thereby changing to a tetragonal $(\mathrm{T})$ phase. Schematic textures with twin formation and phase changes in the $a b$ plane film are proposed and illustrated in Fig. $1 \mathrm{~g}-\mathrm{i}$, and the crystal lattice constant is calculated based on the diffraction mappings given in Fig. 1f.

The time courses of photocatalytic $\mathrm{H}_{2}$ evolution from various $\mathrm{BiVO}_{4}$ phases under visible-light irradiation are shown in Fig. 2. It clearly shows that monoclinic $\mathrm{BiVO}_{4}$ (1\% W doping) demonstrates the best performance of $\mathrm{H}_{2}$ evolution reactions and possesses 3-4 times higher photocatalytic activity than other phases. It is noteworthy that in previous studies, small-electron polarons can be formed in $\mathrm{BiVO}_{4}$ systems ${ }^{48-51}$; however, due to the poor conductivity and charge recombination centers of these systems, the small-electron polarons cannot be the reason for the high photocatalytic performance of $\mathrm{BiVO}_{4}$ (especially with $1 \% \mathrm{~W}$ doping). 

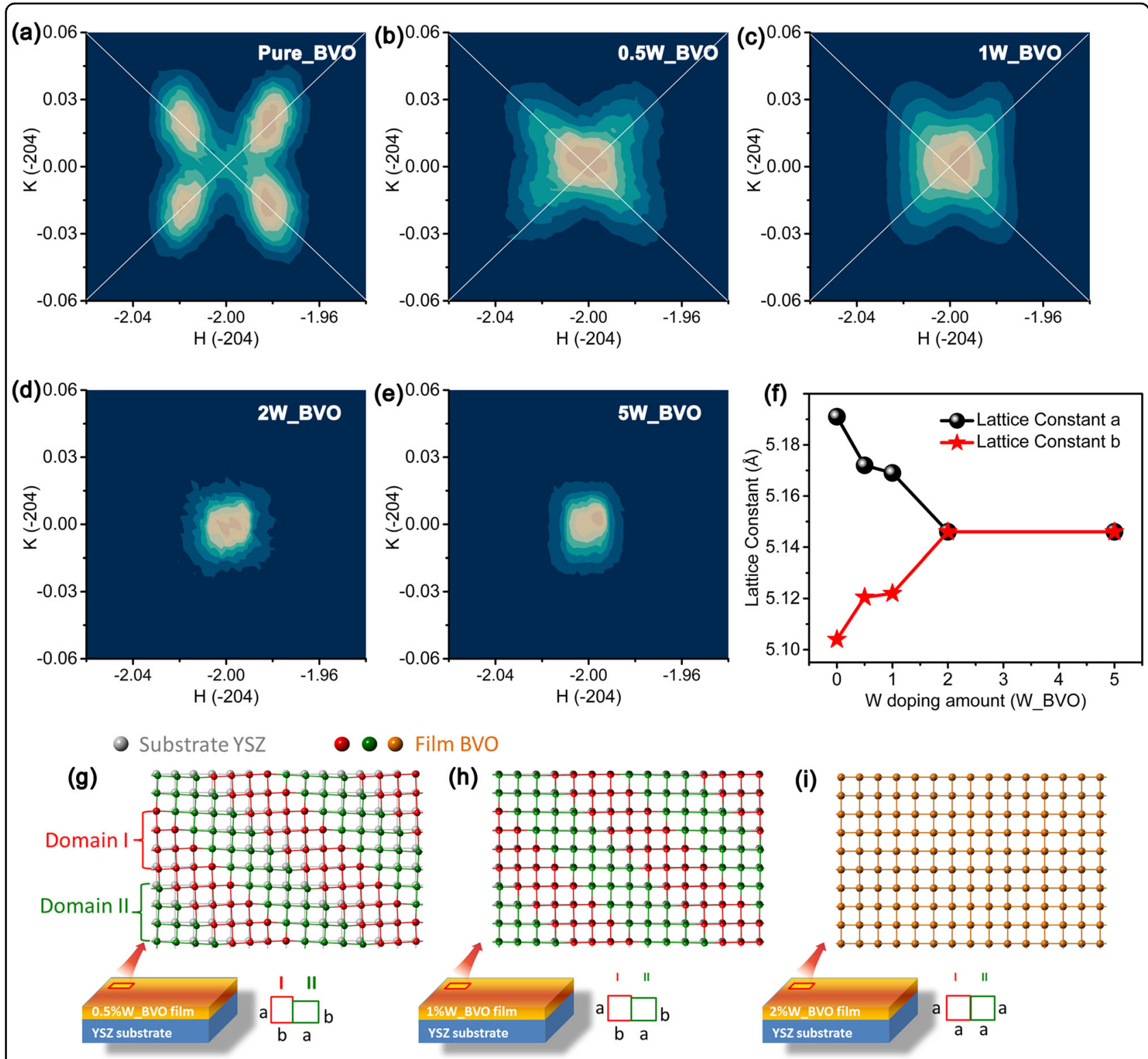

Film BVO

Fig. 1 Crystal structures of $\mathrm{BiVO}_{4}$ with various phases. $\mathrm{RSMs}_{\mathrm{N}}$ of $\mathrm{BiVO}_{4}$ with increasing W doping, where (a), (b), (c), (d) and (e) correspond to pure, $0.5 \%, 1 \%, 2 \%$ and $5 \%$ W-doped $\mathrm{BiVO}_{4}$. The white diagonal thin lines show the in-plane symmetry of the ab lattice. $\mathbf{f}$ Doping dependence of lattice constants. For the tetragonal phase, the ab lattice constants should be the same. $\mathbf{g}$-i Schematically represent how the phases change with increasing W doping. For a pure $\mathrm{BiVO}_{4}$ film, a twin domain with $a \perp b$ is formed under strain due to the substrate, and it shows an orthorhombic phase; when a small amount of $\mathrm{W}$ is introduced, lattice $a$ is no longer perpendicular to lattice $b$ in the twin domain, and the phase changes to monoclinic. However, when the $\mathrm{W}$ doping is increased further, a tetragonal phase of $\mathrm{BiVO}_{4}$ can be obtained.

XAS measurements at both the $\mathrm{V} L$-edge and $\mathrm{O} K$-edge were performed. These measurements are element and site specific and directly probe the electronic structure of the unoccupied states in $\mathrm{BiVO}_{4}$. The XAS results of $\mathrm{W}$-doped $\mathrm{BiVO}_{4}$ are shown in Fig. 3. Interestingly, we observe a strong hole prepeak at $\sim 514.8 \mathrm{eV}$ (Fig. 3a) from the $\mathrm{V} L_{3}$ edge. This hole prepeak, which appears to be a midgap state in SE, as discussed later, is a new many-body large-hole polaron and has the following unique characteristics. First, the hole prepeak $(514.8 \mathrm{eV})$ is dependent on $\mathrm{W}$ doping, with a maximum intensity at $1 \%$ W-doped $\mathrm{BiVO}_{4}$ (Fig. 3c). As W-substituted V in $\mathrm{BiVO}_{4}$ creates vanadium vacancies, the hole prepeak is enhanced by vanadium vacancies. Second, this prepeak is X-ray polarization-dependent, as shown in ref. ${ }^{52}$. This indicates that the prepeak at the $\mathrm{V} L_{3}$-edge not only contains a multiplet feature but also includes a hole state, and this hole state is polarization dependent. This is further 


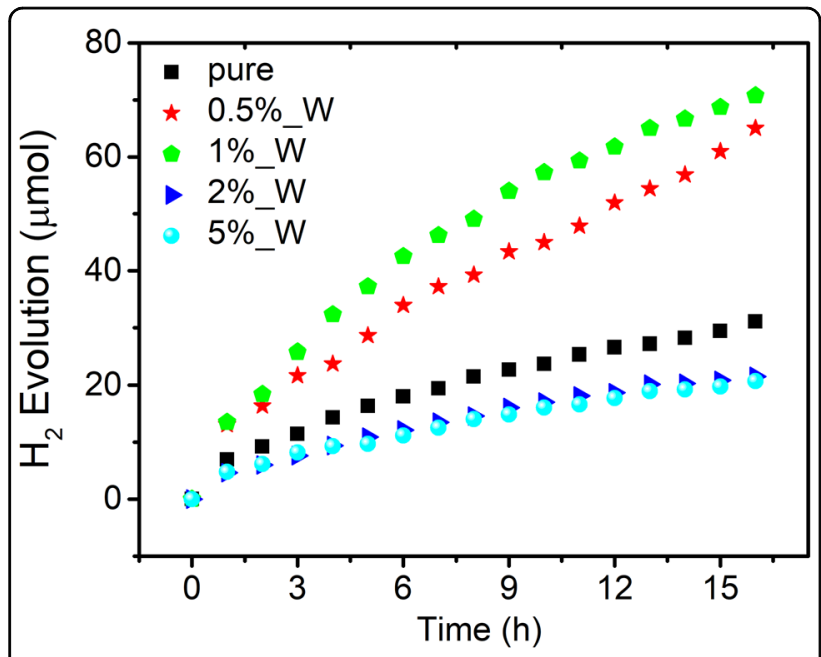

Fig. 2 Photocatalytic water splitting performance. Photocatalytic $\mathrm{H}_{2}$ evolution on $\mathrm{BiVO}_{4}$ with increasing $\mathrm{W}$ doping as a function of time.

confirmed by our theoretical calculations of the XAS and $\mathrm{X}$-ray linear dichroism (XLD) spectra of $\mathrm{V}^{5+}$ using the CTM4XAS package ${ }^{53}$ (Supplementary Fig. S3), in which the multiplet feature near the prepeak is much weaker than the experimental data and does not depend on the X-ray polarization (Supplementary Fig. S4-S5). The main peaks at $\sim 515.8, \sim 516.8$ and $\sim 517.9 \mathrm{eV}$ are the dipoleallowed transitions from $\mathrm{V} 2 p_{3 / 2}$ to the unoccupied $\mathrm{V} 3 d$ orbitals (Fig. 3a). The distinctive absorption peaks exhibit ligand field splitting and dominate the $C B$ states. According to theoretical calculations ${ }^{12,52,54}$, the tetrahedral crystal field splits the $\mathrm{V} L$-edge into two distinct energy states, " $e$ " $\left(d_{x} 2_{-y} 2\right.$ and $\left.d_{z} 2\right)$ and " $t_{2}$ " $\left(d_{x z}, d_{y z}\right.$ and $d_{x y}$ ), and orbital energy splitting provides direct information on the distorted local tetrahedral environments due to $\mathrm{W}$ doping.

To quantitatively investigate orbital energy splitting in detail, we fit the data with Voigt functions (see underneath Fig. 3a, b and Supplementary Fig. S6-S15), and the orbital splitting results are shown in Fig. 3c, d. For lowdoped $\mathrm{BiVO}_{4}$, the splitting energy of $d_{x z}$ and $d_{y z}$, which projects on the $x y$ in-plane, decreases with $\mathrm{W}$ doping, while the energy splitting energy of the out-of-plane shows an opposite trend, indicating a significant lattice distortion. Intriguingly, there is a large jump in the energy splitting between $1 \%$ and $2 \% \mathrm{~W}$-doped $\mathrm{BiVO}_{4}$. This change in electronic structure infers the structural mutability of $\mathrm{BiVO}_{4}$ as a function of $\mathrm{W}$ doping, and this deformation is due to the strain caused by vacancies and doping. Thus, the change in crystal structure induces a large change in the crystal field, which is measured by XAS. Crystal deformation is a prerequisite in creating a polaronic state, such as the one observed here in W-doped $\mathrm{BiVO}_{4}$.
The presence of the large-hole polaron and its hybridizations are further supported by XAS at the $\mathrm{O} K$-edge with an X-ray dipole transition from $\mathrm{O} 1 s$ to $\mathrm{O} 2 p$ hybridized with $\mathrm{V} 3 d$ and $\mathrm{Bi} 6 s p$, as shown in Fig. $3 \mathrm{~b}$. Based on the different hybridized orbital energies between transition metal elements with $\mathrm{O} 2 p$, the spectra are divided into two main parts: low energy $(<531 \mathrm{eV})$ and high energy $(\geq 531 \mathrm{eV})$. The low-energy spectra are deconvoluted into three components, which are mainly attributed to the triplet splitting of $\mathrm{V} 3 d$ orbitals hybridized with $\mathrm{O} 2 p$ orbitals, while the high-energy spectra are attributed to the $\mathrm{O} 2 p$ - $\mathrm{Bi} 6 s p$ hybridizations. The hybridhole states occupy the lowest absorption edge and can be clearly seen from the fitting results (Fig. 3b and Supplementary Figs. S6-S15). It is found that $1 \%$ W-doped $\mathrm{BiVO}_{4}$ exhibits the highest energy splitting of $\mathrm{Bi} 6 \mathrm{p} / \mathrm{s}$ orbitals, while it is smallest for highly doped $\mathrm{BiVO}_{4}$ (Fig. 3d). This salient energy splitting reveals the significant anisotropy of the $\mathrm{Bi} s p$ states as a result of the distortion in the $\mathrm{BiO}_{8}$ dodecahedra of $1 \% \mathrm{~W}$-doped $\mathrm{BiVO}_{4}$. Remarkably, by comparing the pre-edge at both the $\mathrm{V} L$-edge and the $\mathrm{O} K$-edge associated with the large-hole polaron of all the samples, the intensity of the large-hole polaron is highest with $1 \% \mathrm{~W}$-doped $\mathrm{BiVO}_{4}$, which is consistent with the spectroscopic ellipsometry results shown below. This further demonstrates the existence of a large-hole polaron, which is mostly in $\mathrm{O} 2 p$ that is strongly hybridized with V $3 d$ and Bi $6 s p$.

Spectroscopic ellipsometry directly probes the complex dielectric function $\left(\varepsilon(\omega)=\varepsilon_{1}(\omega)+\mathrm{i} \varepsilon_{2}(\omega)\right.$, Fig. $4 \mathrm{a}-\mathrm{c}$ and Supplementary Figs. S16-S21) and is the most direct technique to measure spectral weight transfer (SWT) over a broad energy range ${ }^{55-57}$. SWT is important to reveal electronic structures, correlations, and screenings and shows how the states near the Fermi energy are influenced by higher energy bands ${ }^{52,55-61}$. Based on our electronic structure calculations (the theoretical calculations are shown later in Fig. 5), we divide $\varepsilon_{2}(\omega)$ into four main regions (I, II, III, IV), as shown in Fig. 4a, b. Regions I, II, III and IV of $\varepsilon_{2}$ are separated based on different types of transitions: the spectral weight at region I, which is located at the lowest energy, mainly originates from the Drude response; the spectral weight at region II is mainly from the indirect band-to-band transitions and large-hole polaron; region III denotes the direct band-to-band transitions; and the region IV, which has the highest energy, refers to the higher energy band-to-band transition.

Our main observation is a new midgap state at $\sim 2.63 \mathrm{eV}$ (region II) and is associated with anomalous SWT. From $0 \%$ to $1 \% \mathrm{~W}$ doping, SWT occurs from region III to regions II and IV. After further increasing W doping to $5 \%$, the spectral weight of region II transfers back to regions III and I. The anomalous SWT in such a wide 

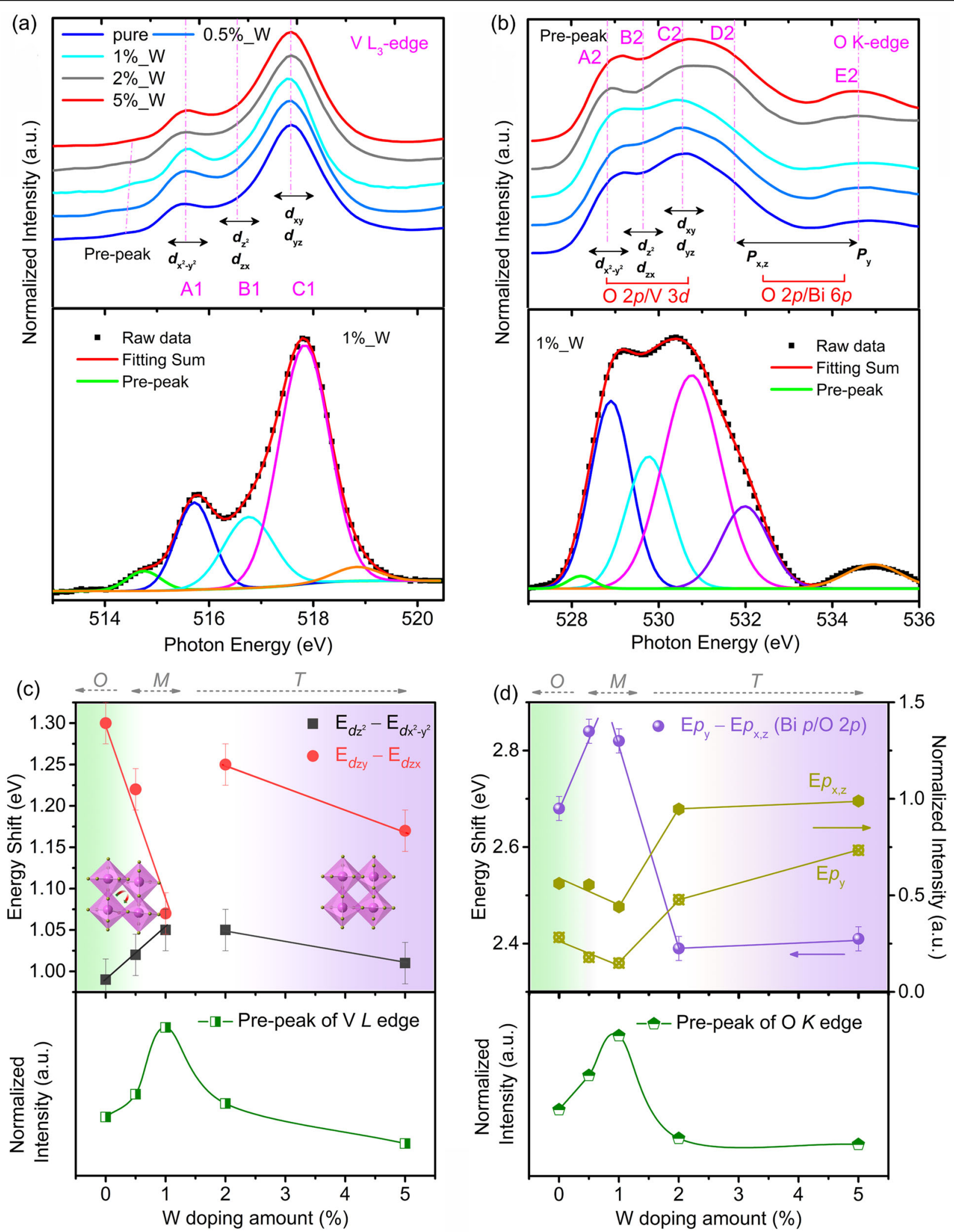

Fig. 3 X-ray absorption spectroscopy at the $\mathbf{V} \mathbf{L}$-edge and $\mathbf{O} \boldsymbol{K}$-edge. Panels $(\mathbf{a}, \mathbf{b})$ are doping-dependent $X A S$ spectra at the $V L_{3}$-edge and $O K$ edge, respectively. The subband orbitals of vanadium are denoted as $A 1, B 1, C 1$, and the metal orbitals hybridized with $O 2 p$ are labeled $A 2$ to E2. The fitting results of the $V L_{3}$-edge and the $O K$-edge using $1 \%$ W-doped $B V O$ as a model are shown underneath. $\mathbf{c}$, $\mathbf{d}$ Show the energy shifts between the subband peaks as a function of $\mathrm{W}$ doping. Underneath (c) and (d) are the prepeak intensities of the $\mathrm{V} L$ - and $\mathrm{O} K$-edges, respectively. 

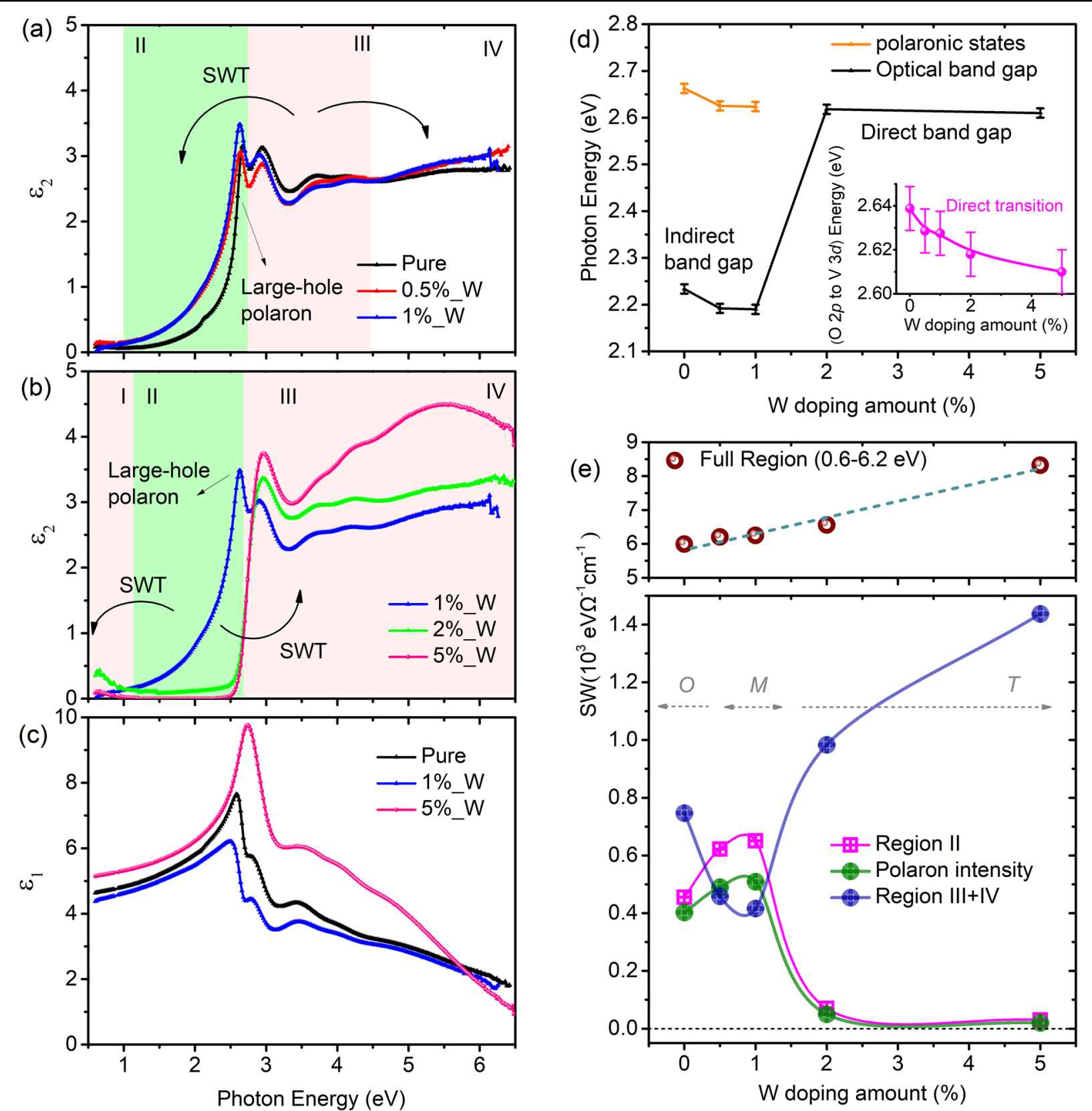

Fig. 4 Complex dielectric functions and spectral weight transfer of $\mathbf{B i V O}_{4}$ films with $\mathbf{W}$ doping. a Imaginary part of the complex dielectric functions, $\varepsilon_{2}(\omega)$, of $\mathrm{BiVO}_{4}$ with $0 \%, 0.5 \%$ and $1 \% \mathrm{~W}$ doping. $\mathbf{b}$ Imaginary part of the complex dielectric functions, $\varepsilon_{2}(\omega)$, of $\mathrm{BiVO}_{4}$ with $1 \%, 2 \%$ and $5 \% \mathrm{~W}$ doping. c Real part of the complex dielectric functions, $\varepsilon_{1}(\omega)$, of $\mathrm{BiVO}_{4}$ with $0 \%, 1$ and $5 \% \mathrm{~W}$ doping. $\mathbf{d}$ Optical energy positions of all transitions at the band edge as a function of $\mathrm{W}$ doping. The inset indicates the $\mathrm{O} 2 p$ to $\vee 3 d$ transition, which is the direct band transition of all samples. e The spectral weight change as a function of $\mathrm{W}$ doping for the polaron peak and regions II and III.

energy range is a direct fingerprint of strong electronic correlations $^{55-57}$. Based on the XAS observations at the $\mathrm{O}$ $K$-and $\mathrm{V} L_{3,2}$-edges and our theoretical calculations, the sharp, midgap state at $\sim 2.63 \mathrm{eV}$ is the large-hole polaronic state. At a lower energy of $\sim 2.2 \mathrm{eV}$, there is another midgap state that is weaker and broader, which is from indirect band transitions (Supplementary Figs. S22-S26).
The SWs in regions III and IV mainly correspond to the optical transitions from the valance band $\mathrm{O} 2 p$ to the conduction bands $\mathrm{V} 3 d$ and $\mathrm{Bi} 6 s p$, respectively. Therefore, the SWT from region III to regions II and IV again demonstrates that the holes are enhanced through electronic correlations and move to a lower hybridized state ( $\mathrm{V} 3 d-\mathrm{O} 2 p$ - Bi $6 s p$ hybridizations) to form the large- 

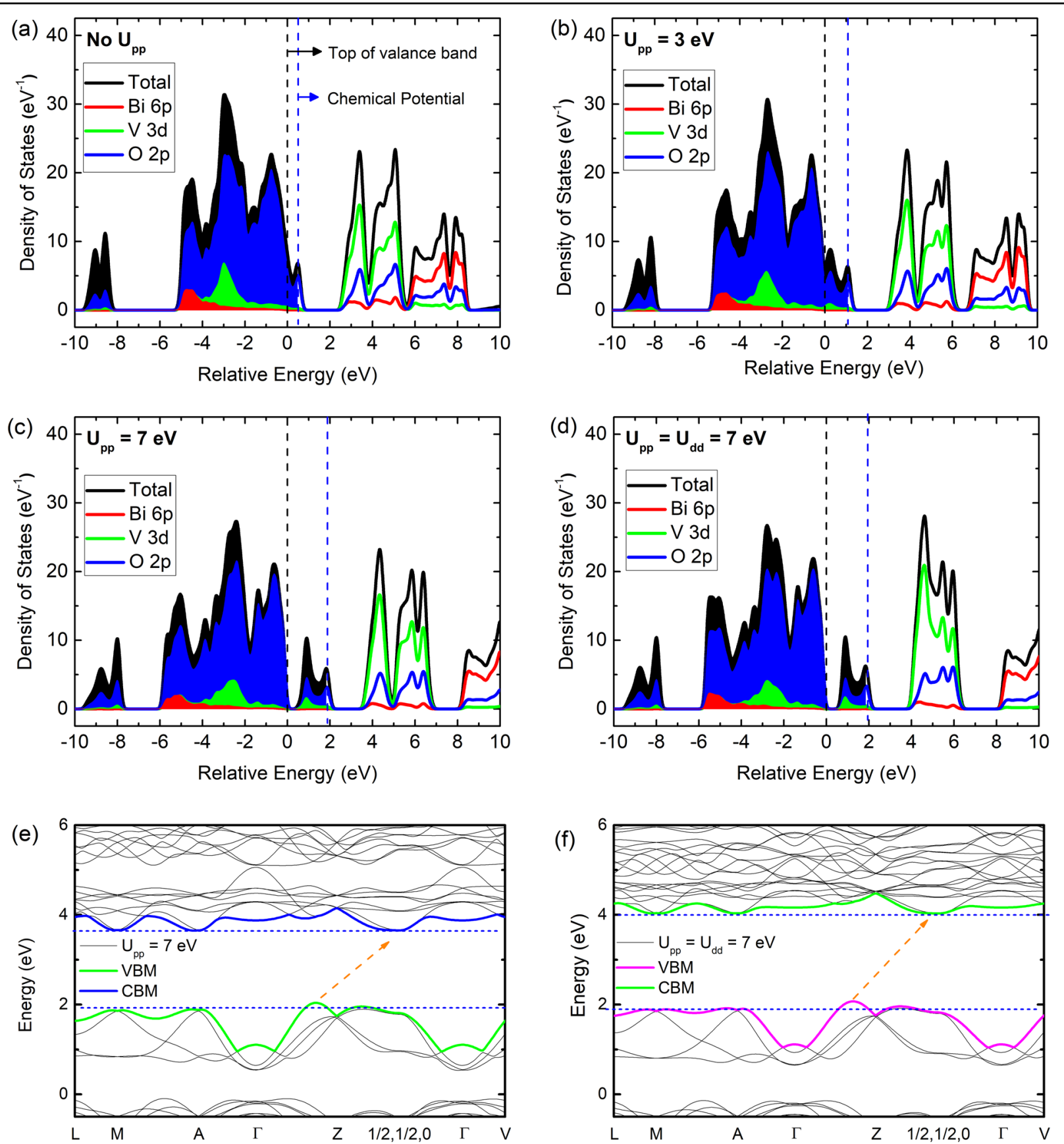

Fig. 5 Electronic structure with electronic correlations studied by first-principles calculations. Density of states of monoclinic $\mathrm{BiVO}_{4} \mathrm{projected}$ onto the $\mathrm{Bi}, \mathrm{O}$ and $\mathrm{V}$ states with on-site Coulomb repulsions: (a) $U_{p p}=U_{d d}=0 \mathrm{eV}$, (b) $\cup_{p p}=3 \mathrm{eV},(\mathbf{c}) U_{p p}=7 \mathrm{eV}$, and (d) $U_{p p}=U_{d d}=7 \mathrm{eV}$. Electronic band structure of monoclinic $\mathrm{BiVO}_{4}$ with different on-site Coulomb repulsions: (e) $\cup_{p p}=7 \mathrm{eV}$ and (f) $\cup_{p p}=\cup_{d d}=7 \mathrm{eV}$.

hole polaron (Fig. S2). All these results indicate that W doping helps to introduce holes and simultaneously enhances electronic correlations to form large-hole polarons in the $\mathrm{BiVO}_{4}$ system. Another important observation is that the SWT also reveals electronic screening, which is dramatically modified by $\mathrm{W}$ doping, as shown in $\varepsilon_{1}(\omega)$ (Fig. 4c). The low values of $\varepsilon_{1}$ in region II show that electronic screening is reinforced in $1 \%$
W-doped BiVO4. Electronic screening is significant and reduces the hole-hole interactions that form the largehole polaron and consequently improves conductivity and carrier mobility, which is required for improved photocatalytic activity.

To quantitatively investigate the SWT and transfer energy as a function of $\mathrm{W}$ doping, we further studied the optical conductivity $\left(\sigma_{1}(\omega)=\varepsilon_{0} \varepsilon_{2}(\omega) \omega\right.$, where $\varepsilon_{0}$ is the 
vacuum permittivity). This is because $\sigma_{1}(\omega)$ obeys the $f$ sum rule (charge conservation, $\int_{0}^{\infty} \sigma_{1}(\omega) d \omega=\pi n e^{2} / 2 m_{e}$, where $n, e$ and $m_{e}$ are the total electron density, elementary charge and electron mass, respectively). The partial SW from $E_{1}$ to $E_{2}$ can be calculated by the following equation:

$$
\mathrm{SW}=\int_{E_{1}}^{E_{2}} \sigma_{1}(\omega) d \omega .
$$

We fit the optical conductivity as a deconvolution of each of the components of the transitions, particularly the polaronic state and the indirect/direct band-to-band transitions (Supplementary Figs. S27-S28). The result shows that the optical bandgap of $\mathrm{BiVO}_{4}$ is altered from indirect to direct with increasing $\mathrm{W}$ doping and that the band gap decreases by almost $0.4 \mathrm{eV}$ for M-phase $\mathrm{BiVO}_{4}$ (Fig. 4d). Importantly, we also found that the excitation energy of the hole polaron is almost identical to the energy of the direct band gap. This result shows that the hole polaron has a small activation energy, further demonstrating that the hole polaron is a large-hole polaron. The SW for the large-hole polaron is shown in Fig. 4e, and it has the same intensity trend as region II, implying that both the large-hole polaron and indirect band gap are influenced by W doping. Note that region II only contains the polaronic state and the indirect band transition. Therefore, we conclude that the large-hole polaron and the indirect band transition originate from electronic correlations and screening and can be modified by $\mathrm{W}$ doping. Finally, we also performed temperaturedependent spectroscopic ellipsometry on $1 \% \mathrm{~W}$-doped $\mathrm{BiVO}_{4}$ (Supplementary Figs. S29-S32). We find that upon cooling, the intensity of the large-hole polaron is enhanced accompanied by spectral weight transfer from high $(>3.2 \mathrm{eV})$ to low energies $(<3.2 \mathrm{eV})$, which further supports the important role of the many-body electronic correlations.

Since the electronic correlations and the crystal structure of $\mathrm{BiVO}_{4}$ change, the electronic band structures also change. Therefore, the previous calculations ${ }^{12,54}$ of the electronic band structure without electronic correlations are not suitable to explain our experimental observations, especially as $\mathrm{W}$ doping introduces strong electronic correlations. Thus, we utilize density functional theory by incorporating on-site Coulomb repulsions on crystal structure $U(\mathrm{DFT}+\mathrm{U})$ and charge transfer multiplex calculations on monoclinic $\mathrm{BiVO}_{4}$. The calculated density of states projected onto $\mathrm{Bi}, \mathrm{O}, \mathrm{V}$ and the electronic band structure are shown in Fig. 5. The strongly correlated nature of electrons and holes can be described by adding a Hubbard-U term to the DFT energy function. We consider the role of electronic correlations of on-site Coulomb repulsion in the $p$ orbital $\left(\mathrm{U}_{p p}\right)$ and $d$ orbital $\left(\mathrm{U}_{d d}\right)$ in the electronic band structures, and a hole is created by removing an electron from the highest occupied orbital. First, we apply $\mathrm{U}_{p p}=0 \mathrm{eV}$ to represent very weak or no electronic correlations (Fig. 5a). Our calculations show that the hole state is located just above the valance band. However, this calculation is not consistent with our experimental results because from our experimental observation, the hole state is located away from the valance band and near the conduction band, as there is no Drude response formed at low energy from the dielectric function (Fig. 4a). Next, we increase on-site Coulomb repulsion to $\mathrm{U}_{p p}=3 \mathrm{eV}$ (Fig. 5b). Interestingly, the on-site Coulomb repulsion in the $p$ orbital pushes holes toward the conduction band. Further increasing to $\mathrm{U}_{p p}=7 \mathrm{eV}$ (Fig. 5c) shows that a hole polaron is created as a midgap state, while the separation between the valence band and conduction band is also enhanced. In addition, the system shows an indirect band gap (Fig. 5e). The hole polaron is mostly $\mathrm{O} 2 p$ hybridized with $\mathrm{V} 3 d$ and $\mathrm{Bi} 6 p$. By considering strong on-site Coulomb repulsion at both the $p$ and $d$ orbitals $\left(\mathrm{U}_{p p}=\mathrm{U}_{d d}=7 \mathrm{eV}\right.$, Fig. $\left.5 \mathrm{~d}\right)$, intriguingly, our theoretical calculations show that the hole polaron (midgap state) is more localized, the separation between the valence band and conduction band is further enhanced, the hybridization between $\mathrm{V} 3 d$ with $\mathrm{O} 2 \mathrm{p}$ and Bi $6 p$ becomes stronger and the indirect band gap is further enhanced. These theoretical calculations with strong on-site Coulomb interactions are consistent with our experimental data. Therefore, with the full support of the theoretical calculations, we find that the role of electronic correlations in the on-site Coulomb interactions of $\mathrm{O} p\left(\mathrm{U}_{p p}\right)$ and $\mathrm{V} d\left(\mathrm{U}_{d d}\right)$ is crucial to the many-body largehole polaron and the electronic structure of monoclinic $\mathrm{BiVO}_{4}$. These new calculations have deepened our understanding of the $\mathrm{BVO}_{4}$ system.

We can now reconcile the large-hole polaron, indirect bandgap and photocatalytic activity in this $\mathrm{BiVO}_{4}$ system with similar thickness (Supplementary Fig. S33). Both the large-hole polaron and the indirect bandgap are significant in $\mathrm{M}-\mathrm{BiVO}_{4}$, while pure $\mathrm{O}-\mathrm{BiVO}_{4}$ shows a large-hole polaron but weak electronic screening and an indirect bandgap. After $\mathrm{W}$ doping, the large hole polaron, indirect bandgap and electronic screening are enhanced the most with the $1 \% \mathrm{~W}$ doping of $\mathrm{M}-\mathrm{BiVO}_{4}$, accompanied by changes in the crystal structure and symmetry. Moreover, the $\mathrm{Bi} 6 s p$ band also dramatically improves. Together, these factors play important roles in the photocatalytic properties of $\mathrm{M}-\mathrm{BiVO}_{4}$ for the following reasons. First, excellent visible-light photocatalytic activity is promoted by the advantage of a narrow bandgap. Second, the photon-induced hole in the $\mathrm{O} 2 p$ band can dynamically relax to a lower energy state, namely, Bi $6 s p$, resulting in highly efficient charge separation and suppression of charge-carrier recombination; therefore, the separated electrons and holes can contribute to the photocatalytic 
activity instead of photoluminescence. Third, due to the small activation energy of the polaronic state in $\mathrm{M}-\mathrm{BiVO}_{4}$, the large-hole polaron favorably forms to determine the transport behavior. Given that the large-hole polaron tends to undergo band-like conduction, it can obviously enhance the conductivity of $\mathrm{M}-\mathrm{BiVO}_{4}$ to improve photocatalytic performance (the comparison of photocatalytic performance with previous work can be seen in Supplementary Fig. S34). With further W doping ( $>1 \% \mathrm{~W}$ doping), both the large-hole polaron and indirect bandgap decrease, accompanied by a reduction in photocatalytic activity. Clearly, the strong electronic correlations and screening induced the large-hole polarons and indirect bandgap, which determined the photocatalytic activity.

\section{Conclusion}

In summary, we observe a new many-body large-hole polaron and indirect bandgap in $\mathrm{W}$-doped $\mathrm{BiVO}_{4}$ films due to strong electronic correlations and screening. The many-body large-hole polarons preserve band-like transport and, in conjunction with the indirect band transition, support fast and highly efficient charge separation, which strongly suppresses charge-carrier recombination, enhances conductivity, and determines photocatalytic efficiency. Our result opens a new path in utilizing the many-body large-hole polaron in transition metal oxides to achieve high photocatalytic activity.

\section{Acknowledgements \\ The authors thank Z. Li, E. Chew, H. Miao, W. Wong, W. Zaw, and C. Lim for their technical support. This work was supported by Ministry of Education of Singapore (MOE) ACRF Tier-2 (MOE2017-T2-1-135, MOE2018-T2-2-117, and MOE2019-T2-1-163), the Singapore National Research Foundation under its Competitive Research Funding (NRF-CRP15-2015-01), NRF - NUS Resilience and Growth Postdoctoral Fellowships (R-144-000-455-281 and R-144-000-459-281) and NUS Core Support (Grant No. C-380-003-003-001). The authors would also like to acknowledge the Singapore Synchrotron Light Source (SSLS) and NUSNNI-NanoCore for providing the facilities necessary for conducting the research. The SSLS is a National Research Infrastructure under the National Research Foundation Singapore.}

\section{Author details}

${ }^{1}$ Department of Physics, National University of Singapore, 117576 Singapore, Singapore. ${ }^{2}$ Singapore Synchrotron Light Source, National University of Singapore, 5 Research Link, 117603 Singapore, Singapore. ${ }^{3}$ Department of Chemistry, National University of Singapore, 3 Science Drive 3, Singapore 117543, Singapore. ${ }^{4}$ Centre for Advanced 2D Materials and Graphene Research Centre, National University of Singapore, 117546 Singapore, Singapore. ${ }^{5}$ NUS Graduate School for Integrative Sciences and Engineering, 117456 Singapore, Singapore. ${ }^{6}$ Institute of Chemical and Engineering Sciences, A*STAR, 1 Pesek Road, Jurong Island, Singapore 627833, Singapore. ${ }^{7}$ Department of Materials Science and Engineering, National University of Singapore, Singapore 117575, Singapore

\section{Author contributions}

X.C., L.M. and C.L. contributed equally to this work. X.C., K.P.L., T.V.V. and A.R. conceived the idea. L.M. and H.K.J. prepared the samples. X.C. and A.R. performed the spectroscopic ellipsometry measurements and analysis. X.C., A. R. and X.Y. performed the $X$-ray absorption measurements and analysis. X.C. and P.Y. performed the XRD measurements. C.L. and K.P.L. performed the photocatalytic experiments. A.D.F., M.A.N. and A.R. did the first-principles calculations. X.C. and A.R. comprehensively analyzed all data and wrote the paper with input from all other coauthors. K.P.L., T.V.V. and A.R. initiated and led the project.

\section{Conflict of interest}

The authors declare no competing interests.

\section{Publisher's note}

Springer Nature remains neutral with regard to jurisdictional claims in published maps and institutional affiliations.

Supplementary information The online version contains supplementary material available at https://doi.org/10.1038/s41427-022-00364-w.

Received: 18 June 2021 Revised: 3 October 2021 Accepted: 7 December 2021.

Published online: 4 March 2022

\section{References}

1. Landau, L. On the motion of electrons in a crystal lattice. Phys. Z. Sowjetunion 3, 664 (1933)

2. Pekar, S. Autolocalization of the electron in a dielectric inertially polarizing medium. Zh. Eksp. Teor. Fiz. 16, 335-40 (1946).

3. Zhao, G. M., Hunt, M., Keller, H. \& Müller, K. Evidence for polaronic supercarriers in the copper oxide superconductors $\mathrm{La}_{2-x} \mathrm{Sr}_{x} \mathrm{CuO}_{4}$. Nature 385, 236 (1997).

4. Peng, $Y$. et al. Disappearance of nodal gap across the insulator-superconductor transition in a copper-oxide superconductor. Nat. Commun. 4, 2459 (2013).

5. Sous, J., Chakraborty, M., Krems, R. V. \& Berciu, M. Light bipolarons stabilized by Peierls electron-phonon coupling. Phys. Rev. Lett. 121, 247001 (2018).

6. Park, J.-H. et al. Direct evidence for a half-metallic ferromagnet. Nature $\mathbf{3 9 2}$, 794 (1998).

7. Jaramillo, R., Ha, S. D., Silevitch, D. \& Ramanathan, S. Origins of bad-metal conductivity and the insulator-metal transition in the rare-earth nickelates. Nat. Phys. 10, 304 (2014).

8. Nie, W. et al. Light-activated photocurrent degradation and self-healing in perovskite solar cells. Nat. Commun. 7, 11574 (2016).

9. Miyata, K. \& Zhu, X.-Y. Ferroelectric large polarons. Nat. Mater. 17, 379 (2018).

10. Walsh, A. et al. Band edge electronic structure of BiVO4: elucidating the role of the Bi s and V d orbitals. Chem. Mater. 21, 547-51 (2009).

11. Park, H. S. et al. Factors in the metal doping of $\mathrm{BiVO}_{4}$ for improved photoelectrocatalytic activity as studied by scanning electrochemical microscopy and first-principles density-functional calculation. J. Phys. Chem. C. 115, 17870-9 (2011).

12. Kweon, K. E. \& Hwang, G. S. Structural phase-dependent hole localization and transport in bismuth vanadate. Phys. Rev. B 87, 205202 (2013).

13. Khaselev, O.\& Turner, J. A. A monolithic photovoltaic-photoelectrochemical device for hydrogen production via water splitting. Science 280, 425-7 (1998).

14. Zou, Z., Ye, J., Sayama, K. \& Arakawa, H. Direct splitting of water under visible light irradiation with an oxide semiconductor photocatalyst. Nature 414, 625 (2001).

15. Khan, S. U., Al-Shahry, M. \& Ingler, W. B. Efficient photochemical water splitting by a chemically modified $\mathrm{n}-\mathrm{TiO}_{2}$. Science $\mathbf{2 9 7}, 2243-5$ (2002).

16. Maeda, K. et al. Photocatalyst releasing hydrogen from water. Nature 440, 295 (2006).

17. Reece, S. Y. et al. Wireless solar water splitting using silicon-based semiconductors and earth-abundant catalysts. Science 334, 645-8 (2011).

18. Dotan, $\mathrm{H}$. et al. Resonant light trapping in ultrathin films for water splitting. Nat. Mater. 12, 158 (2013).

19. Kim, T. W. \& Choi, K.-S. Nanoporous $\mathrm{BiVO}_{4}$ photoanodes with dual-layer oxygen evolution catalysts for solar water splitting. Science 343, 990-4 (2014).

20. Kibsgaard, J. \& Chorkendorff, I. Considerations for the scaling-up of water splitting catalysts. Nat. Energy 4, 430-3 (2019).

21. Roger, I., Shipman, M. A. \& Symes, M. D. Earth-abundant catalysts for electrochemical and photoelectrochemical water splitting. Nat. Rev. Chem. 1, 0003 (2017).

22. Lee, B.-H. et al. Reversible and cooperative photoactivation of single-atom Cu/ $\mathrm{TiO}_{2}$ photocatalysts. Nat. Mater. 18, 620 (2019). 
23. Kudo, A., Omori, K. \& Kato, H. A novel aqueous process for preparation of crystal form-controlled and highly crystalline $\mathrm{BiVO}_{4}$ powder from layered vanadates at room temperature and its photocatalytic and photophysical properties. J. Am. Chem. Soc. 121, 11459-67 (1999).

24. Takata, $T$. et al. Photocatalytic water splitting with a quantum efficiency of almost unity. Nature 581, 411-4 (2020)

25. Sivula, K. \& Van De Krol, R. Semiconducting materials for photoelectrochemical energy conversion. Nat. Rev. Mater. 1, 15010 (2016).

26. Emin, D. Polarons (Cambridge University Press, 2012).

27. Yu, J. \& Kudo, A. Effects of structural variation on the photocatalytic performance of hydrothermally synthesized BiVO4. Adv. Funct. Mater. 16, 2163-9 (2006).

28. Li, R. et al. Spatial separation of photogenerated electrons and holes among $\{010\}$ and $\{110\}$ crystal facets of $\mathrm{BiVO}_{4}$. Nat. Commun. 4, 1432 (2013).

29. Abdi, F. F. et al. Efficient solar water splitting by enhanced charge separation in a bismuth vanadate-silicon tandem photoelectrode. Nat. Commun. 4, 2195 (2013).

30. Lee, D. K. \& Choi, K.-S. Enhancing long-term photostability of BiVO 4 photoanodes for solar water splitting by tuning electrolyte composition. Nat. Energy 3, 53 (2018)

31. Wu, F. \& Ping, Y. Combining Landau-Zener theory and kinetic Monte Carlo sampling for small polaron mobility of doped $\mathrm{BaVO}_{4}$ from first-principles. J. Mater. Chem. A 6, 20025-36 (2018).

32. Mohamed, $\mathrm{M}$. et al. The electronic structure and the formation of polarons in Mo-doped BiVO 4 measured by angle-resolved photoemission spectroscopy. RSC Adv. 9, 15606-14 (2019).

33. Eichhorn, J. et al. Revealing nanoscale chemical heterogeneities in poly-

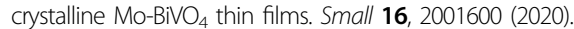

34. Zhong, D. K., Choi, S. \& Gamelin, D. R. Near-complete suppression of surface recombination in solar photoelectrolysis by "Co-Pi" catalyst-modified W: BiVO, J. Am. Chem. Soc. 133, 18370-7 (2011).

35. Ye, K.-H. et al. Enhancing photoelectrochemical water splitting by combining work function tuning and heterojunction engineering. Nat. Commun. 10, 3687 (2019).

36. Kweon, K. E. \& Hwang, G. S. Surface structure and hole localization in bismuth vanadate: a first principles study. Appl. Phys. Lett. 103, 131603 (2013).

37. Murphy, A. et al. Efficiency of solar water splitting using semiconductor electrodes. Int. J. Hydrog. Energy 31, 1999-2017 (2006).

38. Kim, T. W., Ping, Y., Galli, G. A. \& Choi, K.-S. Simultaneous enhancements in photon absorption and charge transport of bismuth vanadate photoanodes for solar water splitting. Nat. Commun. 6, 8769 (2015).

39. Yang, $\mathrm{Y}$. et al. A simple flame strategy for constructing W-doped $\mathrm{BiVO}_{4}$ photoanodes with enhanced photoelectrochemical water splitting. Int. J. Energy Res. 44, 10821-31 (2020).

40. Lamers, M. et al. Formation and suppression of defects during heat treatment of BiVO 4 photoanodes for solar water splitting. J. Mater. Chem. A 6, 18694-700 (2018).

41. Rusydi, A. et al. Cationic-vacancy-induced room-temperature ferromagnetism in transparent, conducting anatase $\mathrm{Ti}_{1-x} \mathrm{Ta}_{x} \mathrm{O}_{2}(x \sim 0.05)$. Thin Films. Philos. Trans. Roy. Soc. Lond. A: Math. Phys. Eng. Sci. 370, 4927-43 (2012).
42. Qi, D. C. et al. Cationic vacancies and anomalous spectral-weight transfer in $\mathrm{Ti}_{1-x} \mathrm{Ta}_{x} \mathrm{O}_{2}$ thin films studied via polarization-dependent near-edge x-ray absorption fine structure spectroscopy. Phys. Rev. B 87, 245201 (2013).

43. Giannozzi, P. et al. QUANTUM ESPRESSO: a modular and open-source software project for quantum simulations of materials. J. Phys.: Condens. Matter 21, 395502 (2009).

44. Hamann, D. Optimized norm-conserving Vanderbilt pseudopotentials. Phys. Rev. B 88, 085117 (2013).

45. Anisimov, V. I., Zaanen, J. \& Andersen, O. K. Band theory and Mott insulators: Hubbard U instead of Stoner I. Phys. Rev. B 44, 943 (1991).

46. Anisimov, V. I. et al. Density-functional theory and $\mathrm{NiO}$ photoemission spectra. Phys. Rev. B 48, 16929 (1993).

47. Liechtenstein, A., Anisimov, V. I. \& Zaanen, J. Density-functional theory and strong interactions: Orbital ordering in Mott-Hubbard insulators. Phys. Rev. B 52, R5467 (1995).

48. Jovic, V. et al. Soft X-ray spectroscopic studies of the electronic structure of M: $\mathrm{BiVO}_{4}(\mathrm{M}=\mathrm{Mo}, \mathrm{W})$ single crystals. J. Mater. Chem. A 3, 23743-53 (2015).

49. Favaro, M. et al. Chemical, structural, and electronic characterization of the (010) surface of single crystalline bismuth vanadate. J. Phys. Chem. C. 123, 8347-59 (2018).

50. Eichhorn, J. et al. Nanoscale imaging of charge carrier transport in water splitting photoanodes. Nat. Commun. 9, 2597 (2018).

51. Kweon, K. E. et al. Electron small polarons and their transport in bismuth vanadate: a first principles study. Phys. Chem. Chem. Phys. 17, 256-60 (2015).

52. Chi, $X$ et al. Oxygen electronic screening and hybridization determining the insulator-metal transition of $\mathrm{Eu}_{1-x} \mathrm{Ba}_{\mathbf{x}} \mathrm{TiO}_{3}$. Phys. Rev. B 98, 085152 (2018).

53. Stavitski, E. \& De Groot, F. M. The CTM4XAS program for EELS and XAS spectral shape analysis of transition metal L edges. Micron 41, 687-94 (2010).

54. Kweon, K. E. \& Hwang, G. S. Hybrid density functional study of the structural, bonding, and electronic properties of bismuth vanadate. Phys. Rev. B 86 165209 (2012).

55. Rusydi, A. et al. Metal-insulator transition in manganites: changes in optical conductivity up to $22 \mathrm{eV}$. Phys. Rev. B 78, 125110 (2008).

56. Asmara, $\mathrm{T}$. et al. Mechanisms of charge transfer and redistribution in $\mathrm{LaAlO}_{3} /$ $\mathrm{SrTiO}_{3}$ revealed by high-energy optical conductivity. Nat. Commun. 5, 3663 (2014).

57. Asmara, T. C. et al. Tunable and low-loss correlated plasmons in Mott-like insulating oxides. Nat. Commun. 8, 15271 (2017)

58. Eskes, H., Meinders, M. \& Sawatzky, G. Anomalous transfer of spectral weight in doped strongly correlated systems. Phys. Rev. Lett. 67, 1035 (1991).

59. Ohta, Y. et al. Evolution of the in-gap state in high-T c cuprates. Phys. Rev. B 46, 14022 (1992).

60. Chi, $X$. et al. Elucidating surface and bulk emission in 3D hybrid organic-inorganic lead bromide perovskites. Adv. Opt. Mater. 6, 1800470 (2018).

61. Chi, X. et al. Unusual hole and electron midgap states and orbital reconstructions induced huge Ferroelectric Tunneling Electroresistance in $\mathrm{BaTiO}_{3} /$ $\mathrm{SrTiO}_{3}$. Nano Lett. 20, 1101 (2020). 